Original Research Paper

\title{
Effects of Flaxseed Supplementation on Lipid Metabolism, Oxidative Balance and Genetic Damage in Goats
}

\author{
${ }^{1}$ Maria Eugenia Cecchini, ${ }^{1}$ Dardo Roma, ${ }^{1}$ Franco Magnago, \\ ${ }^{2}$ María Laura Vilchez, ${ }^{2}$ María Cristina Varea, ${ }^{1}$ María Elena Torreta, \\ ${ }^{2}$ Delia Elba Aiassa and ${ }^{1}$ Fernando Javier Mañas \\ ${ }^{I}$ Facultad de Agronomía y Veterinaria. Universidad Nacional de Río Cuarto. \\ Ruta 36, km 601. Río Cuarto, Provincia de Córdoba. Argentina \\ ${ }^{2}$ Facultad de Ciencias Exactas, Físico-Químicas y Naturales, \\ Universidad Nacional de Río Cuarto. Ruta 36, km 601. Río Cuarto, Provincia de Córdoba. Argentina
}

Article history

Received: 01-02-2018

Revised: 03-04-2018

Accepted: 15-05-2018

Corresponding Author:

Fernando Mañas

Department of Animal Clinic,

National University of Río

Cuarto. Route 36, km 601, Río

Cuarto (5800), Córdoba,

Argentina

E-mail: fmanas@ayv.unrc.edu.ar

\begin{abstract}
Epidemiological evidence indicates that flaxseed reduces oxidative stress and cholesterol levels in blood. In this study we evaluated the lipid profile, oxidation of plasma lipids and genetic damage in goats fed on a diet supplemented with flaxseed. Thirteen adult male goats were split in two experimental groups; one of them was fed on a conventional diet of alfalfa and ground corn and the other group was fed on the same diet supplemented with $5 \%$ of flaxseed. Blood samples were obtained every 7 days to quantify the Thiobarbituric Acid-Reactive Substances (TBARs), total cholesterol, HDL, LDL and triacylglycerols. Additionally, 3 blood samples and 3 oral mucosa samples were taken to each animal every 15 days to perform comet assay and micronucleus test. Flaxseed supplementation produces a remarkable antioxidant effect in plasma in a three months period that could explain the antigenotoxic effect determined through both micronucleus test and comet assay. In addition, we also found a reduction of LDL/HDL ratio and cholesterol levels in animals supplemented with flaxseed.
\end{abstract}

Keywords: Flaxseed, Goats, DNA Protection, Antioxidant, Lipid Profile

\section{Introduction}

Flaxseed is known as a rich source of polyunsaturated fatty acids, dietary fiber and lignans. In vitro studies showed that flaxseed protein hydrolysates have antioxidant and anti-inflammatory activity, as well as cholesterol-lowering ability (Silva et al., 2013). It has a high oil content $(32-45 \%)$, of which $51-55 \%$ is Alpha-Linolenic Acid (ALA, n-3) and $15-18 \%$ is Linoleic Acid (LA, n-6) (Guarda et al., 2016).

Alpha-linolenic acid (ALA) is an essential fatty acid associated, by some researchers, with a decrease of triacylglycerol levels, blood pressure and specific coronary heart diseases (Bloedon and Szapary, 2004). The beneficial effects on cardiovascular health due to ALA may be the result of a drop in plasma levels of triacylglycerols and LDL cholesterol, an increase in HDL cholesterol, a decrease in blood pressure and platelet aggregation and a low incidence of arrhythmias (Valenzuela et al., 2011). Moreover, a decrease in LDL/HDL ratio could also be beneficial due to the potential antioxidant effect of HDL, which could lead to a reduction of LDL oxidation (Assmann and Gotto, 2004).

Some studies have shown that feeding cows, pigs and poultry with flaxseed increases the $\omega-3$ LCPUFA levels of dairy products and foodstuffs derived from these animals. Also, human consumption of such products contributes to maintain high $\omega-3$ LCPUFA levels in red blood cells (Legrand et al., 2010). In addition, other authors have demonstrated that introducing flaxseed in livestock diet reduces $\omega-6$ LCPUFA/ $\omega-3$ LCPUFA ratio in their food stuff products. Moreover, they affirm that is possible for humans to maintain the plasmatic levels of $\omega-3$ LCPUFA without fish intake and through the consumption of animals fed with flaxseed (Weill et al., 2002).

Flaxseed is also a rich source of Secoisolariciresinol Diglucoside lignan (SDG), a precursor of mammalian lignans enterodiol and enterolactone, containing 75-800 times more SDG than other food materials ( $\mathrm{Li}$ et al., 2012). SDG is a phytoestrogen that is transformed in enterolactone and enterodiol by action of gastric acid and bacterial glucosidase in digestive tract. This 
transformation also occurs in ruminant digestive tract, including goat livestock (Zhou et al., 2009).

Both plant lignans and mammalian lignan metabolites are biologically active. In fact, one of their main properties is the high antioxidant activity, related with the suppression of oxidative conditions due to ROS, which produces a DNA protector effect in mammalian cells (Rajesha et al., 2006; Rubilar et al., 2010). It has been proved that certain lignans have the ability to scavenge free radicals, particularly hydroxyl groups. Furthermore, it has been suggested that this molecules have even more antioxidant activity than vitamin E (Prasad, 1997).

The overproduction of Reactive Oxygen Species (ROS) can be implicated in DNA damage. As a result of the ROS attack to the DNA, permanent changes in the genetic material are the first step in the processes of mutagenesis, carcinogenesis and aging. ROS can cause single and double strand DNA breaks, crossovers and changes in purines, pyrimidines, or deoxyribose (Beuret et al., 2005; Astiz et al., 2009). Oxidative stress is accepted as a critical pathophysiological mechanism in different frequent human pathologies, including cancer. In fact ROS can cause protein, lipid DNA damage malignant tumors often show increased levels of DNA base oxidation and mutations (Mena et al., 2009). Flaxseed is among the foods with highest capacity to prevent spontaneous chromosomal damage in mice (Toure and Xueming, 2010). According to the available literature, the ability of flaxseed to prevent genetic damage is related with the antioxidant activity, mainly due to its high content of lignans. Thus, many authors associate the antioxidant activity of lignans with the capacity to prevent neoplastic diseases (Prasad, 1997; Kitts et al., 1999).

Therefore, in the present study we intend to evaluate the lipid profile, oxidation of plasma lipids and genetic damage in goats fed on a diet supplemented with flaxseed compared with goats fed on a conventional diet.

\section{Materials and Methods}

\section{Experimental Site}

The experiment was carried out in accordance with the U.K. Animals (Scientific Procedures) Act, 1986 and associated guidelines, EU Directive 2010/63/EU for animal experiments. The animals used in this study were thirteen adult (14 months of age) male Anglo-Nubian goats, housed in facilities of the Agronomy and Veterinary faculty, National University of Río Cuarto.

\section{Animals and their Management}

The animals were randomly split in two experimental groups; one of them ( 7 goats) was fed on a conventional and controlled diet (not ad libitum) of alfalfa hay (80\%), ground corn $(19.5 \%)$ and a vitamin-mineral premix $(0.5 \%)$ according to the NRC guidelines (2007), the other group (6 goats) was fed on the same diet supplemented with $5 \%$ of flaxseed. The premix contained: Calcium 10\%, Phosphorus $8.00 \%$, Salt 42.00\%, Potassium 0.10\%, Magnesium 1.00\%, Copper 1,750.00 ppm, Selenium 25.00 ppm, Zinc 7,500.00 ppm, Vitamin A 140,000 IU/lb, Vitamin D3 11,000 IU/lb, Vitamin E $750 \mathrm{IU} / \mathrm{lb}$. Flax seeds did not receive any treatment before being administered to the animals. In both cases, natural conditions of photoperiod were kept and water was available ad libitum to the goats.

\section{Blood Sampling}

Heparinized blood samples were obtained every 7 days to quantify the Thiobarbituric Acid-Reactive Substances (TBARs) and to determine the lipid and biochemical profile through measuring total cholesterol, HDL, LDL and triacylglycerols. To quantify the thiobarbituric acid-reactive substances, thirteen samples per individual were taken, collecting the first sample 7 days before the beginning of flaxseed supplementation. To determine lipid profile, 12 blood samples were taken, of which, the first one was collected 7 days after the study started. Additionally, blood samples were taken to each animal every 15 days to perform comet assay, collecting the samples 60 days after starting the treatment and completing a total of 3 blood samples to each animal. Simultaneously 3 oral mucosa samples were obtained to carry out micronucleus test. In all cases, blood samples were collected by jugular vein puncture under aseptic conditions, $5 \mathrm{~mL}$ samples were taken for weekly determinations of oxidative stress and lipid profile and $1 \mathrm{~mL}$ samples for the comet assay.

\section{Methodology used in the Research}

\section{Thiobarbituric Acid-Reactive Substances (TBARs) Analysis}

Each blood sample was centrifuged (3000 RPM) and the removed plasma was preserved at $-20^{\circ} \mathrm{C}$ for further analysis. Quantification of thiobarbituric acid-reactive substances was carried out following the protocol originally described by Buege and Aust (1978), modified by Marcincak et al. (2003). Malondialdehyde (MDA) was used as a reference substance for TBARs determination. All determinations were performed in duplicated and each sample was quantified in triplicate. Results are expressed in terms of nmol MDA/g protein.

\section{Micronucleus Test in Oral Mucosa}

The assay followed the guidelines proposed by Thomas et al. (2009) with some modifications. The oral epithelium samples were collected using sterile swabs, rinsing the oral cavity with distilled water previously. The cells were fixed with a solution of Methanol: Glacial 
Acetic Acid (3:1). Smears were colored with 10\% Giemsa solution for 10 minutes and, finally, they were observed with optical microscope. A total of 1000 epithelial cells were analyzed searching for micronucleated cells and nuclear abnormalities.

\section{Single-Cell Gel Electrophoresis (Comet Assay)}

The comet assay was performed according to the protocol proposed by Singh et al. (1988), with slight modifications. Volumes of $50 \mu \mathrm{L}$ of blood from each mouse were added to $100 \mu \mathrm{L}$ of $0.75 \%$ LMP agarose at $37^{\circ} \mathrm{C}$. The mixtures were layered onto slides pre-coated with $0.75 \%$ NMP agarose and covered with a coverslip. The slides were immersed in a lysis solution $(2.5 \mathrm{M}$ $\mathrm{NaCl}, 100 \mathrm{mM}$ EDTA, $10 \mathrm{mM}$ Tris ( $\mathrm{pH} 10)$, supplemented with $1 \%$ Triton X-100 and 10\% DMSO (just before use) for at least $1 \mathrm{~h}$, right after the slides immersed in a freshly prepared alkaline buffer $\mathrm{pH}>3$ and electrophoresis was conducted for $30 \mathrm{~min}$. The slides were fixed in absolute ethanol, stained with ethidium bromide scored using a fluorescent microscope. From each treatment, images from 100 "nucleoids" were captured with a camera attached to the fluorescent microscope and linked to the Comet Score 1.5 software. Highly damaged cells were not included in the scoring (clouds were not analyzed). Tail Moment (TM) was used to estimate DNA damage (arbitrary units).

\section{Biochemical Analysis}

Commercial kits (Wiener lab, Argentina) were used to determine total cholesterol, LDL, HDL and triacylglycerols in goat plasma. Volumes of 10-200 $\mu \mathrm{L}$ were mixed with the pertinent reactive at $37^{\circ} \mathrm{C}$ and homogenized in the cuvette. The absorbance was measured at $505 \mathrm{~nm}$ and all the results were expressed as $\mathrm{mg} / \mathrm{dL}$ of plasma.

\section{Statistical Analysis}

The Kolmogorov-Smirnov test was performed to verify whether the results follow a normal distribution. Student $t$ test was used to examine possible statistically significant differences between the groups. To determine whether exist differences between groups through time, ANOVA test was applied followed by Dunnett's test as a subsequent analysis. In all cases, the level of significance was set at $\mathrm{p}<0.05$.

\section{Results}

In this study, a total of 156 samples were taken to evaluate plasma lipoperoxidation in both groups (with and without flaxseed supplementation). No significant differences were observed in goats fed with conventional diet, lipoperoxidation levels were relatively constant through the entire experience. Regarding the group supplemented with flaxseed, lipoperoxidation levels showed a statistically significant decrease from week 7 onwards (ANOVA, Dunnett's test; Fig. 1).

Determination of genetic damage through micronucleus test in oral mucosa resulted in the identification of normal epithelial cells, micronucleated cells and nuclear alterations such as nucleoplasmic bridges (Fig. 2).

Number of micronucleus and nucleoplasmic bridges found in oral mucosa of animals with and without flaxseed supplementation are presented in Table 1 .

The lasts two samplings showed statistically significant differences between animals put on a conventional diet and the ones put on a diet supplemented with flaxseed at $\mathrm{p} \leq 0.05$ and $\mathrm{p} \leq 0.001$ in the second and third sampling, respectively (Student $t$ test). As can be observed, in the group of animals fed with flaxseed, a decrease of $1000 \%$ in the number of micronucleus was detected in the second sampling $(0.16$ $\mathrm{MN})$ regarding the first one $(1.6 \mathrm{MN})$. The same decrease of $1000 \%$ in the number of micronucleus was observed in the third sampling regarding the second one.

Tail moment values for each group and each sampling are presented in Table 2.

Statistically significant differences were observed between the group put on a conventional diet and the group supplemented with flaxseed over the 3 samplings. Figure 3 shows the average levels of cholesterol and LDL/HDL ratio in both groups throughout the treatment period.

Statistically significant differences were found in cholesterol levels and LDL/HDL ratio $(p \leq 0,001$ in both cases)between the groups fed with a conventional diet and the ones fed on a diet supplemented with flaxseed. No significant differences were found in triacylglycerols levels between both groups.

Table 1: Number of Micronucleus (MN) and other nuclear alterations (nucleoplasmic bridges, NPB), in animals fed with a conventional diet and supplemented with flaxseed. Mean \pm standard deviation statistics are shown

\begin{tabular}{|c|c|c|c|c|c|c|}
\hline & Sampling & & & & & \\
\hline & $1^{\circ} \mathrm{SAMPLING}$ & & $2^{\circ} \mathrm{SAMPLING}$ & & $3^{\circ} \mathrm{SAMPLING}$ & \\
\hline & $\mathrm{MN}$ & NPB & $\mathrm{MN}$ & NPB & $\mathrm{MN}$ & NPB \\
\hline$\overline{C D}$ & $2.0 \pm 1.9$ & $6.1 \pm 3.7$ & $1.8 \pm .3$ & $9.6 \pm 5.9$ & $1.6 \pm 0.8$ & $13.6 \pm 4.9$ \\
\hline WF & $1.6 \pm 1.0$ & $4.7 \pm 2.9$ & $0.16 \pm 0.4^{*}$ & $7.83 \pm 4.3$ & $0.016 \pm 0.04 * * *$ & $10.7 \pm 4.4$ \\
\hline
\end{tabular}

${ }^{*} \mathrm{p} \leq 0.05$. ${ }^{* * *} \mathrm{p} \leq 0.001$. Student $\mathrm{t}$ test. $\mathrm{C} / \mathrm{D}$ : conventional diet. W/F: With flaxseed 
Table 2: Levels of DNA damage (tail moment) in animals of both groups through 3 consecutive samplings. Mean \pm standard deviation statistics are shown

\begin{tabular}{llll}
\hline Group & First sampling & Second sampling & Third sampling \\
\hline Conventional diet & $9293.2 \pm 1490.0$ & $9432.3 \pm 1251.0$ & $8638.0 \pm 1849.0$ \\
Supplementation with flaxseed & $6382.7 \pm 1051.0 *$ & $5048.1 \pm 914.0 * *$ & $3792.9 \pm 845.0 * * *$ \\
\hline
\end{tabular}

${ }^{*} \mathrm{p} \leq 0.05 ; * * \mathrm{p} \leq 0.01 ; * * * \mathrm{p} \leq 0.001 ;$ Student $\mathrm{t}$ test

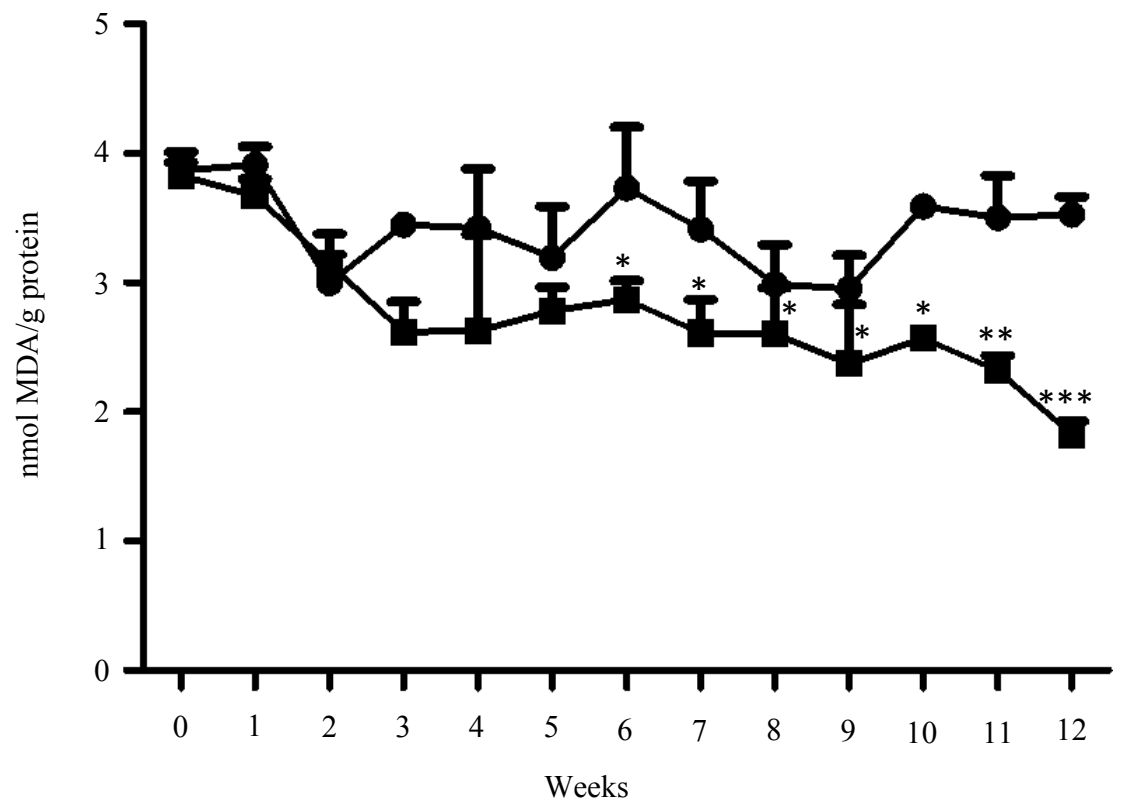

Fig. 1: Levels of MDA in plasma of goats fed on a conventional diet $(\bullet)$ or supplemented with flaxseed (5\%) (घ) for a period of 12 weeks. Results are expressed as mean values \pm SEM. ${ }^{*} p \leq 0.05 .{ }^{* *} p \leq 0.01 . * * * p \leq 0.001$, Dunnett's test

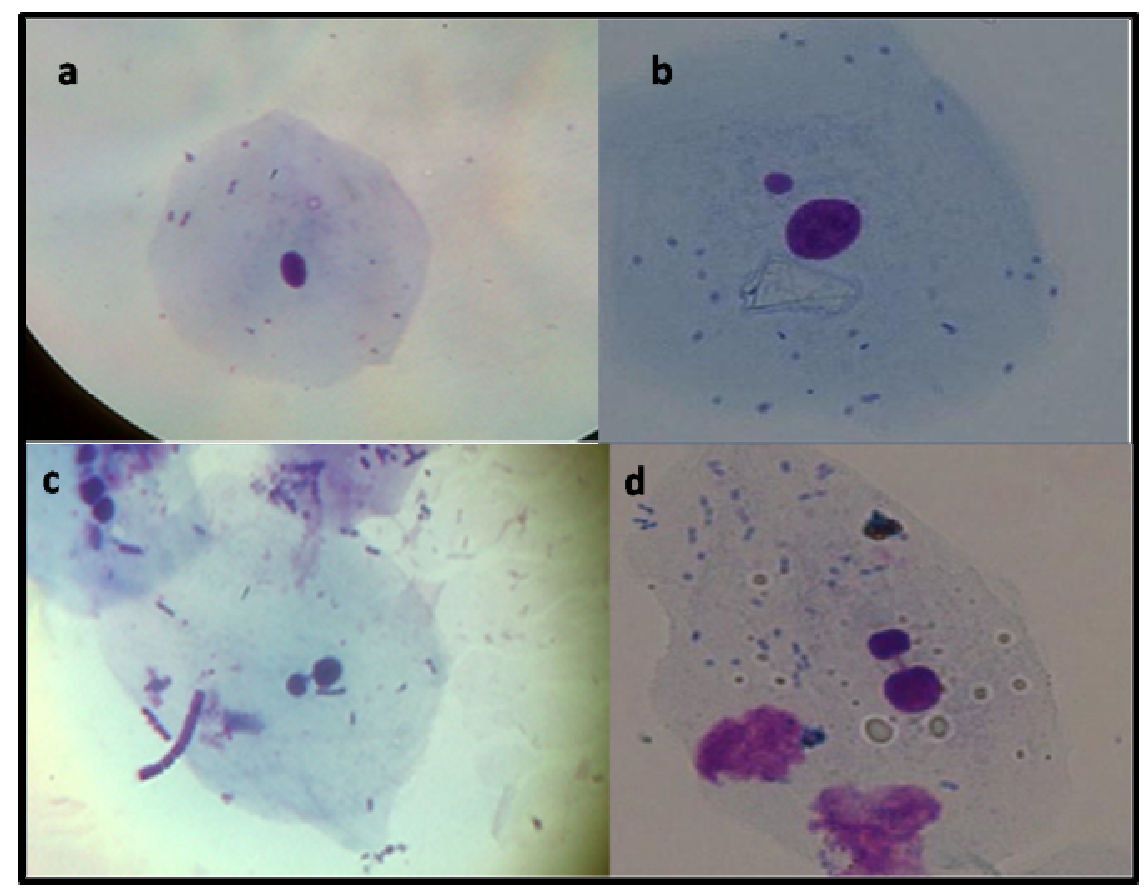

Fig. 2: (a) Oral mucosal epithelial cell of male goat. (b) Oral mucosal epithelial cell of male goat with micronucleus. (c) and (d) Images of oral mucosal epithelial cell of goats with nucleoplasmic bridge. Optical microscope 100X. Giemsa staining $10 \%$ 

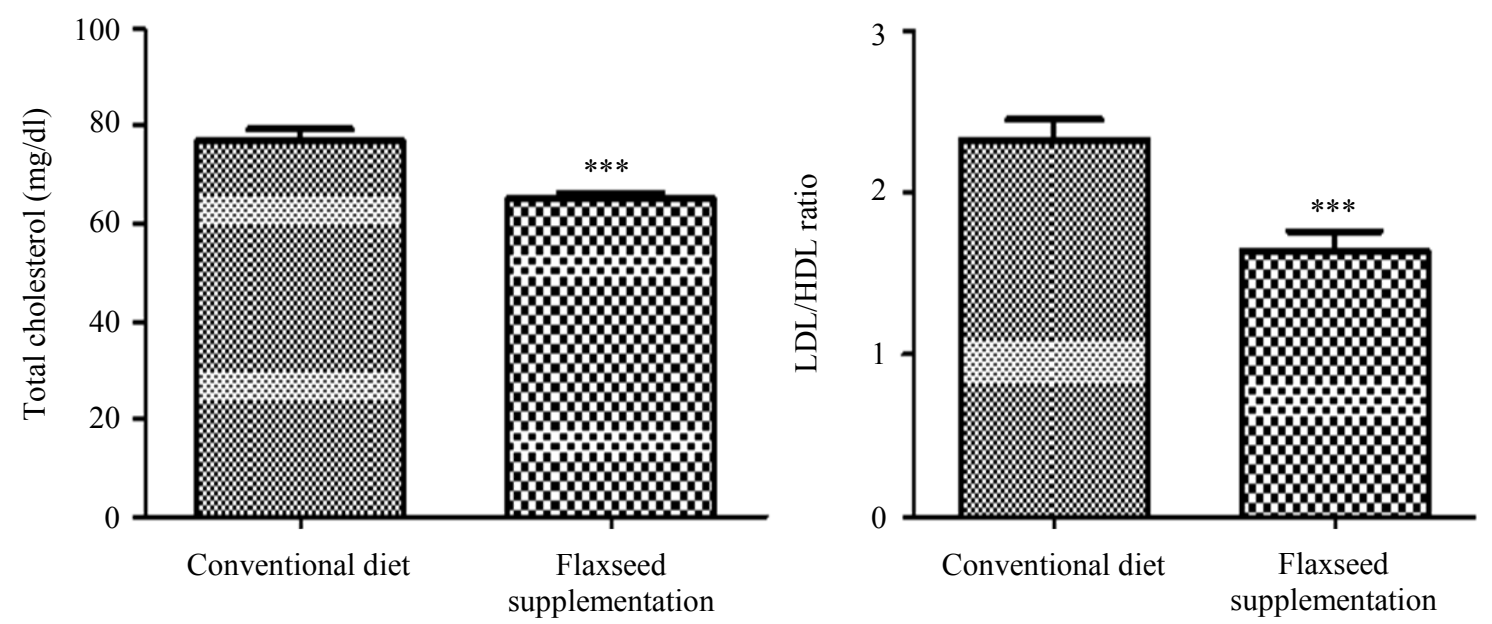

Fig. 3: Left: Total cholesterol levels $(\mathrm{mg} / \mathrm{dL})$ in animals fed with a conventional diet and fed on a diet supplemented with flaxseed. Right: LDL/HDL ratio (mg/dL) in animals fed with a conventional diet and fed on a diet supplemented with flaxseed. Results are expressed as mean values \pm SEM. ${ }^{* * *} \mathrm{p} \leq 0.001$, Student $t$ test.

\section{Discussion}

The results of this study suggest that consumption of flaxseed reduces MDA concentration and, therefore, it may have a beneficial effect over oxidative balance by decreasing lipoperoxidation. As shown in Fig. 1, at the end of the treatment the group that received a diet supplemented with flaxseed has a reduction of around $50 \%$ in peroxidation levels when compared to the group that received a conventional diet, suggesting an antioxidant effect that could protect against the pathogenesis of several diseases. Besides the differences found between the groups, it was observed an effect related with the exposure time in goats supplemented with flaxseed. In this group, plasma MDA levels drop the first few weeks of treatment before stabilizing up to week 7 of supplementation, in which the differences when compared to the beginning of the experience (week 0 ) are statistically significant (ANOVA, Dunnett's test). At week 6 of treatment, a reduction of $25 \%$ in lipoperoxidation levels can be observed compared to levels obtained at first stage of this study. By the end of week 12 , peroxidation levels were $53 \%$ lower than initial values, indicating a time-dependent effect of flaxseed.

Rajesha et al. (2006) proved an antioxidant effect in animals supplemented with different flaxseed concentrations and exposed to carbon tetrachloride as a pro-oxidant agent. These authors found a decrease in Catalase, Peroxidase and Superoxide enzymes reaching 40, $42123 \%$, respectively. Moreover, treatment with carbon tetrachloride increased $20 \%$ lipoperoxidation levels compared to control group. Supplementation with $5 \%$ flaxseed, i.e., the same concentration used in the present study, restored levels of antioxidant enzymes and simultaneously, lipoperoxidation values decreased when compared to the group exposed to carbon tetrachloride. According the authors, these results clearly confirm that supplementation with flaxseed (at 5 and $10 \%$ ) protects animals against oxidative alterations caused by exposition to toxic substances (Rajesha et al., 2006).

When animals are supplemented with lignans, their antioxidant activity can be spread to several organs and tissues. Kinetic studies in rodents treated with orally administered tritiated SDG show significant amounts of radioactivity in plasma, intestinal content and different organs such as liver, kidney, mammary gland, uterus and ovaries, suggesting that lignans reach all these tissues from the intestinal lumen (Kitts et al., 1999). Quantifiable amounts of intracellular Enterodiol (ED) have been found in human epithelial cells after 4 hours of incubation, also, Enterolactone (EL) conjugation by the same cells above mentioned were observed in in vitro studies after identical period of time. Therefore, these results indicate that antioxidant activity of mammalian lignans could provide protection against oxidative stress both intra and extracellularly (Hu et al., 2007).

Their ability to act as antioxidant molecules in mammalian cells interior is related with the aptitude of such compounds to protect DNA integrity. Kitts et al. (1999) stated that mammalian lignans (EL and ED) were effectives to avoid DNA damage caused by reactive oxygen species at concentrations of $10-1000 \mu \mathrm{M}$ (reachable in human colonic lumen). The results obtained in the present study indicate that flaxseed supplementation has a protective effect over genetic material of goat epithelial cells. A statistically significant decrease in the number of micronucleated cells was observed in the animals fed on a diet supplemented with flaxseed when compared to the group put on a conventional diet, beginning with the second sampling (Table 1). The protective effect over the genetic material 
in animals supplemented with flaxseed was evident from week 10 of exposure, suggesting a cumulative effect of the treatment, which coincides with the antioxidant activity, observed through TBARs assay. When comparing both tests, it is noted that TBARs and micronucleus values present statistically significant differences at the same moment. The same applies for comet assay, in which was possible to observe that DNA damage levels decrease over time, in the three sampling performed (Table 2). Considering the results obtained in TBARs assay, regarding the protective effects against oxidative damage over time the effects over genetic material of each applied technique, it could be assumed that flaxseed antioxidant activity generates an antigenotoxic effect, implying a preventive effect against the development of neoplastic diseases.

Kitts et al. (1999) report that flaxseed lignans have the power to avoid the deleterious effects that hydroxyl radicals cause over DNA. Therefore, the authors affirm that these compounds have a protective ability over genetic material, mainly triggered by a strong antioxidant effect. In the same way, $\mathrm{Hu}$ et al. (2007) informed about the in vitro protective effect of lignans over DNA exposed to free radicals, demonstrating that flaxseed antioxidant activity is the responsible of the protective effect over the integrity of genetic material.

Regarding the analisis of lipid metabolism, this study proved that supplementation with flaxseed produces alterations on the lipid profile of treated animals (Fig. 3). Cholesterol levels showed a statistically significant decrease in the group of animals treated with $5 \%$ of flaxseed over a three months period. Such reduction in cholesterol levels could be due to the fact that lignan complex isolated from flaxseed contains 3-hydroxy3methylglutaric acid, a strong hypocholesterolemic agent Prasad, 2005). Other authors found a reduction of cholesterol values in rats orally treated with flaxseed lignan concentrate over a period of seven weeks (Zanwar et al., 2013). In the same way, Guarda et al. (2016), demonstrated that ingestion of flaxseed oil by lactating rats reduces cholesterol levels in milk, suggesting also a hypocholesterolemic effect of that product. In the present study, it was observed that animals supplemented with flaxseed presented a statistically significant reductionof LDL/HDL ratio. In animals treated with flaxseed for three months, there was a decrease of LDL levels along with an increase in plama levels of HDL. A drop in LDL concentration implies a benefit regarding the risk to suffer cardiovascular diseases in the future, mainly due to the responsibility of LDL as a major factor in formation of atheromatous plaque on the wall of blood vessels.Furthermore, an increase in HDL levels leads to an opposite effect, transporting lipids from peripheral tissues to liver tissue and, consecuently, minimizing theiraccumulation in blood vessels (Zhao et al., 2004). Available literature, including several studies with an average duration of 8.5 weeks, indicates that supplementation with flaxseed produces a decrease of total cholesterol and plasma LDL levels; however, it does not substantially affect triacylglycerols concentration in blood (Pan et al., 2009). These results coincide with the ones obtained in the present study, in which, statistically significant differences were not found in triacylglycerols levels between the group of animals supplemented with flaxseed and the group fed on a conventional diet.

\section{Conclusion}

Flaxseed supplementation produces a remarkable antioxidant effect in plasma, reducing lipoperoxidation levels about $50 \%$ in a three months period, however, the significative decrease beggins in week seven of treatment. The antioxidant ability of flaxseed leads to an antigenotoxic effect, which is clearly observable from the seventh week of exposure and has a a higher magnitude by the end of treatment.The ability to protect the integrity of genetic material in animal cells could explain the preventive power over the development of neoplasms attributed to flaxseed.Although a decrease in triacylglycerols concentration was not found, animals supplemented with $5 \%$ of flaxseed during a three months period show asignificative hypocholesterolemic effect. Alongside this, the reduction of LDL/HDL ratio implies a double beneficial impact over lipid profile: A decrease in LDL levels, major responsible in thegenesis of atherosclerosis; and an increase in HDL levels, which opposite effects involve a diminution in accumulation and oxidation of LDL, the trigger toformation of atheromatous plaque on the wall of blood vessels. For these reasons, it is possible to affirm that alterations in the lipid profile of animals supplemented with flaxseed represent a reduction in the risk to suffer cardiovascular diseases in the future.

\section{Acknowledgement}

This work was supported by the Science and Technology Secretary of the National University of Rio Cuarto.

\section{Author's Contributions}

Maria Eugenia Cecchini: Blood sampling, comet assay and writing of the manuscript.

Dardo Roma: Thiobarbituric Acid-Reactive substances (TBARs) analysis.

Franco Magnago: Animal feeding, blood sampling and collaboration with TBARs.

María Laura Vilchez: Micronucleus test.

María Cristina Varea: Bichemical analysis.

María Elena Torreta: Experimental design and statistical analysis. 
Delia Elba Aiassa: Capture and analysis of images from the comet assay with software.

Fernando Javier Mañas: Experimental design, collaboration in blood sampling, slides elaboration for comet assay, interpratation of biochemical analysis and writing of biochemical analysis and writing of the manuscritp.

\section{Conflict of Interest Statement}

None of the authors has any financial or personal relationships that could inappropriately influence or bias the content of the paper.

\section{References}

Assmann, G. and A. Gotto, 2004. HDL cholesterol and protective factors in atherosclerosis. J. Am. Heart Assoc., 109: 8-14.

DOI: 10.1161/01.CIR.0000131512.50667.46

Astiz, M., M.J.T. de Alaniz and C.A. Marra, 2009. The impact of simultaneous intoxication with agrochemicals on the antioxidant defense system in rat. Pestic. Biochem. Physiol. 94: 93-99.

DOI: 10.1016/j.pestbp.2009.03.005

Beuret, C.J., F. Zirulnik and M.S. Giménez, 2005. Effect of the herbicide glyphosate on liver lipoperoxidation in pregnant rats and their fetuses. Reprod. Toxicol., 19: 501-504. DOI: 10.1016/j.reprotox.2004.09.009

Bloedon, L.T. and P.O. Szapary, 2004. Flaxseed and cardiovascular risk. Nutr. Rev., 62: 18-27. DOI: $10.1111 / \mathrm{j} .1753-4887.2004 . t b 00002 . x$

Buege, J.A. and S.D. Aust, 1978. Microsomal lipid peroxidation. Methods Enzymol., 52: 302-310. DOI: $10.1016 / \mathrm{S} 0076-6879(78) 52032-6$

Guarda, D.S., E.G. de Moura, J.C. Carvalho, A.M. dos Reis and P.N. Soares et al., 2016. Maternal flaxseed oil intake during lactation changes body fat, inflammatory markers and glucose homeostasis in the adult progeny: Role of gender dimorphism. J. Nutr. Biochem., 35: 74-80. DOI: $10.1016 /$ j.jnutbio.2016.05.011

Hu, C., Y.V. Yuan and D.D. Kitts, 2007. Antioxidant activities of the flaxseed lignan secoisolariciresinol diglucoside, its aglycone secoisolariciresinol and the mammalian lignans enterodiol and enterolactone in vitro. Food Chem. Toxicol., 45: 2219-2227.

Kitts, D.D., Y.V. Yuan, A.N. Wijewickreme and L.U. Thompson, 1999. Antioxidant activity of the flaxseed lignan secoisolariciresinol diglycoside and its mammalian lignan metabolites enterodiol and enterolactone. Mol. Cell. Biochem., 202: 91-100.
Legrand, P., E. Beauchamp, D. Catheline, F. Pédrono and V. Rioux, 2010. Short chain saturated fatty acids decrease circulating cholesterol and increase tissue pufa content in the rat. Lipids, 45: 975-986. DOI: $10.1007 / \mathrm{s} 11745-010-3481-5$

Li, M.X., H.Y. Zhu, D.H. Yang, X.Q. Ma and C.Z. Wang et al., 2012. Production of secoisolariciresinol from defatted flaxseed by bacterial biotransformation. J. Applied Microbiol., 113: 1352-1361. DOI: 10.1111/j.1365-2672.2012.05436.x

Marcincak, S., J. Sokol, P. Turek, H. Rozanska and Z. Dicakova et al., 2003. Comparative evaluation of analytical techniques to quantify malondialdehyde in broiler meat. Bull. Vet. Inst. Pulawy, 47: 491-496.

Mena, S., A. Ortega and J.M. Estrela, 2009. Oxidative stress in environmental-induced carcinogenesis. Mutat. Res., 674: 36-44.

DOI: $10.1016 /$ j.mrgentox.2008.09.017

NRC, 2007. Nutrient Requirements of Small Ruminants: Sheep, Goats, Cervids New World Camelids. 1st Edn., National Academies Press, Washington, D.C., ISBN-10: 0309102138, pp: 362.

Pan, A., D. Yu, W. Demark-Wahnefried, O.H. Franco and X. Lin, 2009. Meta-analysis of the effects of flaxseed interventions on blood lipids. Am. J. Clin. Nutr., 90: 288-297. DOI: 10.3945 /ajcn.2009.27469

Prasad, K., 1997. Hydroxyl radical-scavenging property of Secoisolariciresinol Diglucoside (SDG) isolated from flax-seed. Mol. Cell. Biochem., 168: 117-123. DOI: $10.1023 / \mathrm{A}: 1006847310741$

Prasad, K., 2005. Hypocholesterolemic and antiatherosclerotic effect of flax lignan complex isolated from flaxseed. Atherosclerosis, 179: 269-275. DOI: 10.1016/j.atherosclerosis.2004.11.012

Rajesha, J., K.N.C. Murthy, M.K. Kumar, B. Madhusudhan and G.A. Ravishankar, 2006. Antioxidant potentials of flaxseed by in vivo model. J. Agric. Food Chem., 54: 3794-3799. DOI: 10.1021/jf053048a

Rubilar, M., C. Gutiérrez, M. Verdugo, C. Shene and J. Sineiro, 2010. Flaxseed as a source of functional ingredients. J. Soil Sci. Plant Nutr., 10: 373-377.

Silva, F.G.D., Y. O'Callagahan, N.M. O'Brien and F.M. Netto, 2013. Antioxidant capacity of flaxseed products: the effect of in vitro digestion. Plant Foods Hum. Nutr., 68: 24-30. DOI: $10.1007 / \mathrm{s} 11130-012-0329-6$

Singh, N.P., M.T. McCoy, R.R. Tice and E.L. Schneider, 1988. A simple technique for quantitation of low levels of DNA damage in individual cells. Exp. Cell Res., 175: 184-191. DOI: $10.1016 / 0014-4827(88) 90265-0$ 
Thomas, P., N. Holland, C. Bolognesi, M. Kirsch-Volders and S. Bonassi et al., 2009. Buccal micronucleus cytome assay. Nat. Protoc., 4: 825-837.

DOI: $10.1038 /$ nprot.2009.53

Toure, A. and X. Xueming, 2010. Flaxseed lignans: Source, biosynthesis, metabolism, antioxidant activity, bio-active components health benefit. Compr. Rev. Food Sci. Food Saf., 9: 261-269. DOI: 10.1111/j.1541-4337.2009.00105.x

Valenzuela, R., G. Tapia, M. González and A. Valenzuela, 2011. Omega-3 fatty acids (EPA and DHA) and its application in diverse clinical situations. Rev. Chil. Nutr., 38: 356-367.

Weill, P., B. Schmitt, G. Chesneau, N. Daniel and F. Safraou et al., 2002. Effects of introducing linseed in livestock diet on blood fatty acid composition of consumers of animal products. Ann. Nutr. Metab., 46: 182-191.

DOI: $10.1159 / 000065405$
Zanwar, A.A., M.V. Hegde and S.L. Bodhankar, 2013. Protective role of concomitant administration of flax lignan concentrate and omega-3-fatty acid on myocardial damage in doxorubicin-induced cardiotoxicity. Food Sci. Hum. Wellness, 2: 29-38. DOI: 10.1016/j.fshw.2013.01.002

Zhao, G., T.D. Etherton, K.R. Martin, S.G. West and P.J. Gillies et al., 2004. Dietary $\alpha$-linolenic acid reduces inflammatory and lipid cardiovascular risk factors in hypercholesterolemic men and women. J. Nutrit., 134: 2991-2997. DOI: $10.1093 / \mathrm{jn} / 134.11 .2991$

Zhou, W., G. Wang, Z. Han, W. Yao and W. Zhu, 2009. Metabolism of flaxseed lignans in the rumen and its impact on ruminal metabolism and flora. Ani. Feed Sci. Technol., 150: 18-26.

DOI: 10.1016/j.anifeedsci.2008.07.006 